\section{Steroid responsive Meningitis Arteritis and concurrent Osteoma cutis in a dog}

\section{James Barton, Daniela Alder, Filippo De Bellis}

Southern Counties Veterinary Specialists, Ringwood, United Kingdom

\section{OBJECTIVES}

Osteoma cutis refers to heterotropic ossification within the skin. Whilst veterinary reports are few, a number of cases suggest an association of osteoma cutis in dogs with chronic glucocorticoid therapy. Steroid Responsive Meningitis Arteritis (SRMA) is an immune mediated inflammatory disorder of the leptomeninges and associated arteries. It is typically seen in young dogs between 6 and 18 months. Patients commonly present with cervical pain and pyrexia. Prednisolone monotherapy is the mainstay of treatment.

We report a case of osteoma cutis in a dog with a concurrent relapse of SRMA. We also report the use of cytarabine as a successful treatment for the SRMA.

\section{METHODS}

A one-year old female neutered Border Collie presented with cervical pain and pyrexia. Clinical examination also revealed multiple hard non-painful plaques beneath the skin between the shoulder blades and base of the tail. Wedge biopsies of the skin lesions and a cisternal CSF tap were taken for histopathology and cytology respectively.

\section{RESULTS}

Cytology of CSF revealed a neutrophilic pleocytosis consistent with SRMA. Histopathology showed the presence of calcified bone within the subcutis.

Given the association within the literature of osteoma cutis and steroid use, the dog was started on intravenous cytarabine infusions. As they were not causing any morbidity, the osteoma cutis lesions were monitored.

After 6 months of follow up the dog is in remission and showing no ill effects from her skin lesions.

\section{STATEMENT (CONCLUSIONS)}

To the best of our knowledge, we report the first case in the literature of cytarabine as an effective monotherapy for SRMA and only the fourth case in the literature of osteoma cutis.

\section{Prevalence of}

neurological disorders in Cavalier King Charles Spaniels in neurology referral

\section{populations}

\section{Katie Brown ${ }^{1}$, Rowena Packer ${ }^{2}$, Holger Volk ${ }^{3}$, Clare Rusbridge ${ }^{1,4}$}

\footnotetext{
University of Surrey, Guildford, United Kingdom The Royal Veterinary Collage, Hertfordshire, United Kingdom The Royal Veterinary College, Hertfordshire, United Kingdom Fitzpatrick Referrals, Godalming, United Kingdom
}

\section{OBJECTIVES}

This study aimed to determine the distribution of clinical presentations and the prevalence of neurological conditions in Cavalier King Charles Spaniels (CKCS). A particular focus was placed upon establishing if syringomyelia was a prevalent disease in CKCS and a frequent diagnosis in referral populations.

\section{METHODS}

Records of 500 CKCS presenting to Fitzpatrick Referrals and the Royal Veterinary College neurology departments over a selected four-year period (September 2013September 2017) were searched. Data was examined to determine the presenting clinical signs and final diagnosis made and analysis was carried out to establish the prevalence and frequency of these.

\section{RESULTS}

The most common clinical presentations of all neurological conditions were behavioural signs of pain (312 cases; $62.4 \%)$, spinal pain $(238 ; 47.6 \%)$, phantom scratching (121; $24.2 \%)$ and gait abnormalities $(90 ; 18.0 \%)$. The most common final diagnosis was syringomyelia (216 affected; $43.2 \%$ prevalence), orthopaedic conditions (74; 14.8\%) and intervertebral disc disease $(60 ; 12.0 \%)$. Other less frequently documented conditions included Chiari-like malformation associated pain $(56 ; 11.2 \%)$, myoclonus (19; $3.8 \%)$, epilepsy $(18 ; 3.6 \%$ and skin disease $(12 ; 2.4 \%)$. 


\section{STATEMENT (CONCLUSIONS)}

This study provides vital information for veterinarians, breeders and owners of CKCS about common presentations of syringomyelia and other neurological diseases to allow for earlier recognition of these potentially painful disorders. It concludes that syringomyelia is the most prevalent neurological disease in referral practices and emphasizes the frequency of pain associated with neurological disorders, but when compared to previous studies, it may be underdiagnosed in first opinion practices.

\section{Rostral skull changes in Cavalier King Charles spaniels with Chiari-like malformation and syringomyelia}

\section{Eleonore Dumas ${ }^{1}$, Susan Penny Knowler ${ }^{1}$, Felicity Stringer ${ }^{2}$, Clare Rusbridge ${ }^{2}$}

1 School of Veterinary Medicine, Faculty of Health \& Medical Sciences, University of Surrey, Guildford, United Kingdom

2 Fitzpatrick Referrals, Godalming, United Kingdom

\section{OBJECTIVES}

Chiari-like malformation (CM) and secondary syringomyelia (SM) have proven associations to multiple morphological traits of the caudal cranium and cervical spine in Cavalier King Charles Spaniels (CKCS). Since brachycephaly increases risk for disease, this study focused on the morphology of the rostral skull and forebrain and aimed to identify physical features associated to development of CM associated pain (CM-P) and SM with a more severe phenotype.

\section{METHODS}

Analysis of 12 measurements ( 11 lines and 1 angle) taken on T2 weighted midsagittal MRI of the skull and cervical spinal cord of 66 CKCS enabled the comparison of 3 phenotypic groups: dogs without SM or CM-P (control group; $n=11$ ), dogs with CM-P only ( $n=15)$, dogs with clinically severe CM/SM (syrinx transverse diameter $\geq 4 \mathrm{~mm}$ and clinical signs relating to $S M ; n=40$ ).

\section{RESULTS}

SM-affected CKCS had an increased height of the cranium $(p=0.028)$ and reduced depth of the stop ( $p=0.006)$ compared to controls. SM-affected CKCS also had a significant reduction in height of the nasal cavity compared to CM-P $(p=0.014)$. There were 2 additional significant traits comparing dogs with and without SM; SM affected dogs had a more ventral orientation of the olfactory bulbs $(p=0.014)$ and shorter distance between basicranium and hard palate $(p=0.031)$.

\section{STATEMENT (CONCLUSIONS)}

Dogs with symptomatic CM/SM are more likely to have brachycephalic features of the rostral skull with "midface" hypoplasia similar to craniosyostosis Crouzan syndrome. This not only enhances our understanding of the disease and "at risk" head conformation but could also impact assessment of MRI and disease diagnosis.

\section{Unusual clinical presentation of dystrophin-deficient feline muscular dystrophy in the UK}

\section{Aldara Eiras-Diaz, Jessica Florey \\ Dick White Referrals, Six Mile Bottom, United Kingdom}

\section{OBJECTIVES}

Dystrophin-deficient feline muscular dystrophy is a rare myopathy caused by absence of dystrophin, a large protein found in cardiac and skeletal muscles. The majority of previously reported cases have presented with generalised muscle hypertrophy and overt gait abnormalities.

\section{METHODS}

An eight month old male neutered domestic short hair was referred for further investigation of a four day history of regurgitation and tongue lesions. Multiple non-painful raised white plaques were visualised on the tongue. Mild pelvic limb stiffness and plantigradism was detected. Cranial nerves were assessed to be normal.

\section{RESULTS}

Moderately increased ALT, AST and markedly raised Creatine Kinase were documented. Thoracic radiographs revealed severe oesophageal dilation, cardiomegaly, scalloped diaphragm and sternal malformation. Tongue lesion histology revealed calcinosis circumscripta. These features raised the suspicion of feline muscular dystrophy. 